\title{
Prediction of giant magneto-impedance effect in amorphous glass-coated micro-wires using artificial neural network
}

Asli Ayten Kaya*

\section{"Correspondence:}

aslitay@uludag.edu.tr

Physics Department, Faculty of Arts

and Sciences, Uludag University,

Gorukle Campus, Bursa, 16059,

Turkey

\begin{abstract}
This paper deals with a prediction of a giant magneto-impedance (GMI) effect on amorphous micro-wires using an artificial neural network (ANN). The prediction model has three hidden layers with fifteen neurons and full connectivity between them. The ANN model is used to predict the GMl effect for $\mathrm{CO}_{70.3} \mathrm{Fe}_{3.7} \mathrm{~B}_{10} \mathrm{Si}_{13} \mathrm{Cr}_{3}$ glass-coated micro-wire. The results show that the ANN model has a $98.99 \%$ correlation with experimental data.
\end{abstract}

Keywords: giant magneto-impedance effect; amorphous micro-wires; modeling; artificial neural network

\section{Introduction}

The origin of the GMI effect is attributed to a combination of the skin effect and magnetic domain behavior in soft ferromagnetic material. The GMI ratios have been calculated as

$$
\frac{\Delta Z}{Z} \%=\frac{Z(H)-Z\left(H_{\max }\right)}{Z\left(H_{\max }\right)} x 100
$$

where $\frac{\Delta Z}{Z}, Z(H)$ and $Z\left(H_{\max }\right)$ are the GMI ratio, magneto impedance at magnetic field $H$, and magneto impedance at maximum magnetic field, respectively. The discovery of the giant magneto-impedance (GMI) effect in soft ferromagnetic amorphous micro-wires makes them very attractive candidate material for making high-performance magnetic sensors [1-6]. The use of such micro-wires could also have implications for the next generation of electronic devices, which will involve increasingly smaller components [7-9]. The GMI effect is termed the giant variation of impedance of a ferromagnetic conductor with an ac current in an applied dc magnetic field [10,11].

Artificial neural networks are increasingly becoming useful in prediction of magnetic performance in electromagnetic devices. The ANN operates like a human brain. ANNs have been applied in many areas (such as electronics, forecasting, banking and aerospace) because of these features. The available ANN software today provides many neural network architectures and learning algorithms, and it also helps users to apply ANN to their specific problems easily $[12,13]$.

In this investigation, the GMI effects are modeled using self-organizing feature map (SOFM) and previous experimental data [11] of $\mathrm{Co}_{70.3} \mathrm{Fe}_{3.7} \mathrm{~B}_{10} \mathrm{Si}_{13} \mathrm{Cr}_{3}$ amorphous glasscoated micro-wire.

@ 2013 Kaya; licensee Springer. This is an Open Access article distributed under the terms of the Creative Commons Attribution License (http://creativecommons.org/licenses/by/2.0), which permits unrestricted use, distribution, and reproduction in any medium, provided the original work is properly cited. 


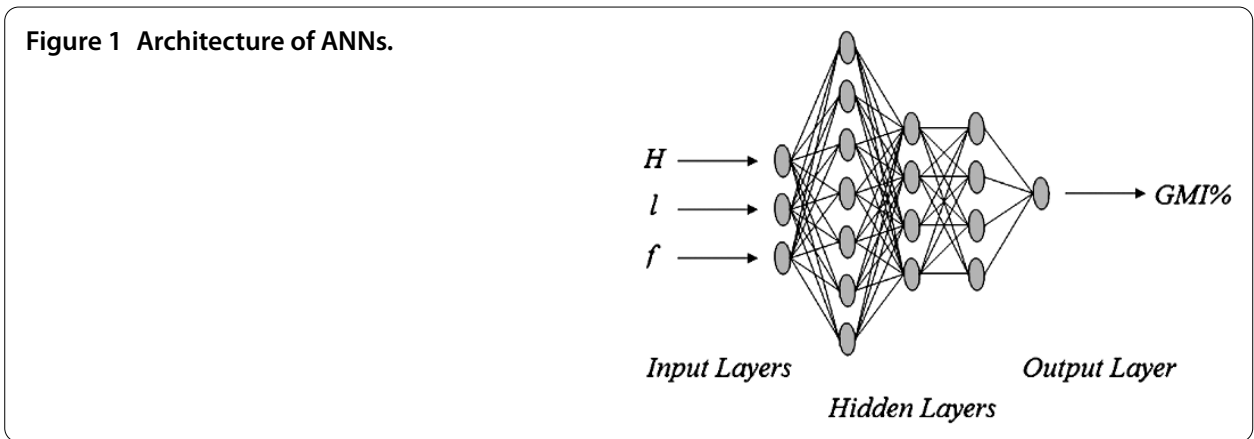

Figure 2 Network outputs plotted against the experimental observations.

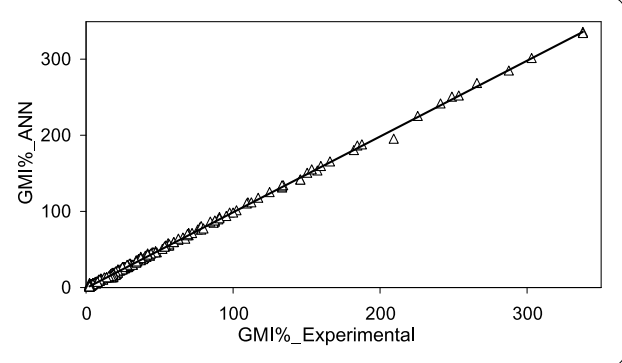

\section{Experimental procedure}

\section{Kohonen neural network}

The ideas of self-organizing feature map (SOFM) are rooted in competitive learning networks. The SOFM transforms the input of an arbitrary dimension into a one- or twodimensional discrete map subject to a topological (neighborhood-preserving) constraint. The feature maps are computed using Kohonen unsupervised learning. The output of the SOFM can be used as the input to a supervised classification neural network [14].

\section{ANN model for glass-coated amorphous micro-wire}

The experimental data used in this work were collected in research by Qin [11]. The developed neural network, which has three input neurons, one output neuron, three hidden layers, seven, four and four neurons of hidden layers and full connectivity between neurons is shown in Figure 1.

The input parameters were magnetizing field $(H)$, wire length $(l)$ and frequency $(f)$. A total of 1,200 input vectors obtained from varied samples [11] were available in the training data. The number of hidden layers and neurons in each layer were determined through trial and error to be optimal including different transfer functions such as hyperbolic tangent, sigmoid and hybrid. After the network was trained, a better result was obtained from the network formed by the hyperbolic tangent transfer function in the hidden and output layers. The number of epochs was $10^{6}$ for training.

\section{Results and discussion}

The $\mathrm{Co}_{70.3} \mathrm{Fe}_{3.7} \mathrm{~B}_{10} \mathrm{Si}_{13} \mathrm{Cr}_{3}$ amorphous glass-coated micro-wires [11] have been used for the experimental confirmation of the proposed model. The agreement between the experimental observations and the network output (predictions) for training is shown in Figure 2. As shown in Figure 2, the values obtained through the training of the ANN model are very close to experimental results, indicating a strong correlation between the input 

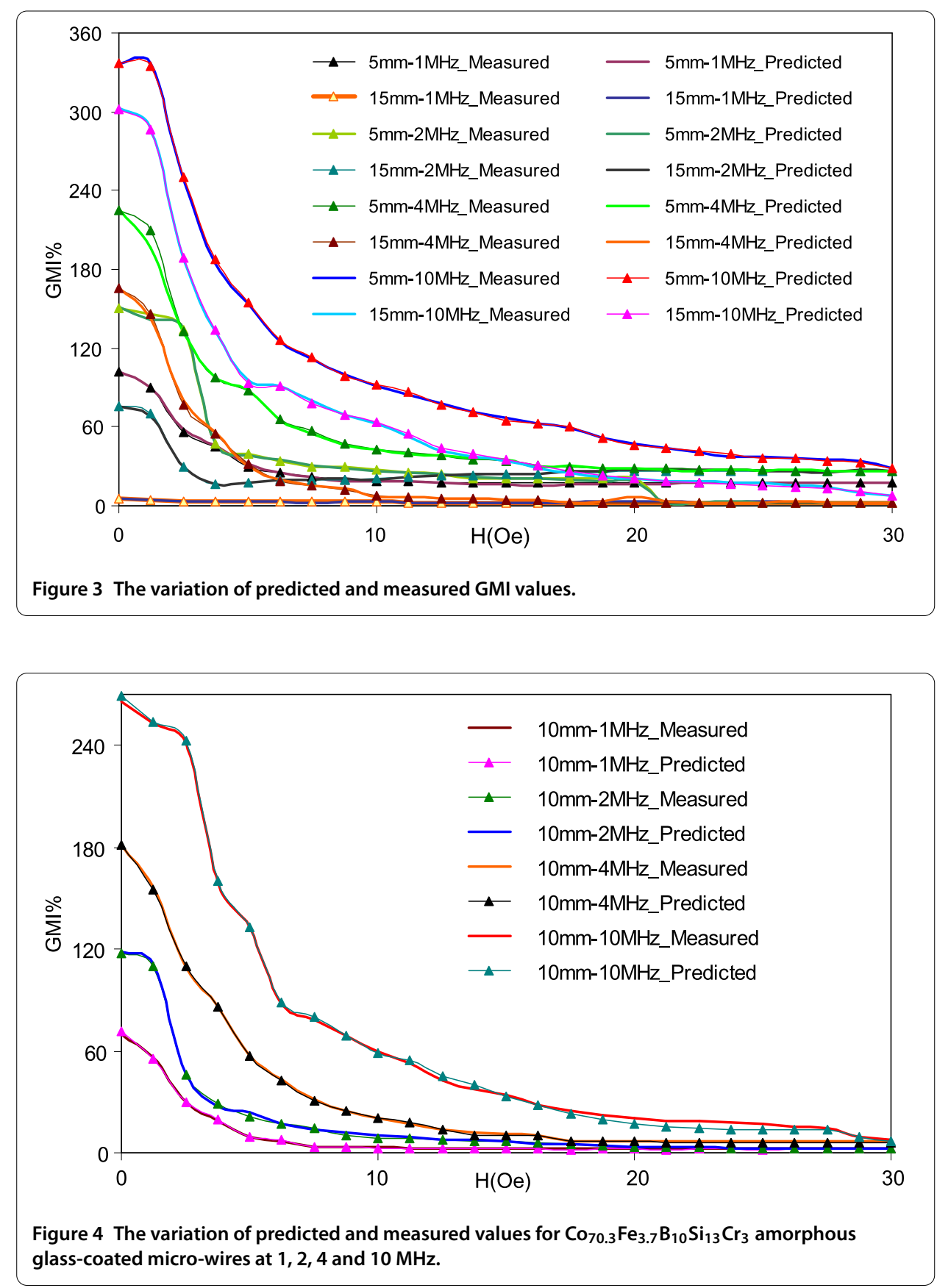

and output parameters of the ANN model. The statistical value of $\mathrm{R}^{2}$ found from ANN training is 0.994 .

Figure 3 shows the variation of the GMI curves obtained from the neural network model and the experimental data. The GMI values achieved from the proposed model are in 99\% agreement with the experimental GMI values. The variation of the GMI curves for untrained data given in Figure 4 and Table 1 also shows a good correlation between measured and predicted results.

All statistical values prove that the proposed ANN model is suitable to predict the GMI values very close to the experimental results. 
Table 1 Comparison of predicted and measured GMI\% values

\begin{tabular}{lcc}
\hline Wire length-frequency & Max GMl\% (measured) & Max GMl\% (predicted) \\
\hline $10 \mathrm{~mm}-1 \mathrm{MHz}$ & 70.00 & 71.62 \\
$10 \mathrm{~mm}-2 \mathrm{MHz}$ & 117.14 & 118.05 \\
$10 \mathrm{~mm}-4 \mathrm{MHz}$ & 181.82 & 181.43 \\
$10 \mathrm{~mm}-10 \mathrm{MHz}$ & 265.63 & 267.29 \\
\hline
\end{tabular}

\section{Conclusions}

The proposed model developed from experimental data obtained previously can be used to predict more easily the GMI curves for $\mathrm{Co}_{70.3} \mathrm{Fe}_{3.7} \mathrm{~B}_{10} \mathrm{Si}_{13} \mathrm{Cr}_{3}$ amorphous glass-coated micro-wires (5, 10 and $15 \mathrm{~mm}$ length) at 1, 2, 4 and $10 \mathrm{MHz}$. According to the results obtained from modeling a neural network, the neural network with the architecture of the ANN method is suitable for predicting the GMI values of Co-based amorphous glasscovered micro-wire. The overall prediction error is about $1 \%$ compared with the measured GMI values.

\section{Competing interests}

The author declares that they have no competing interests.

\section{Acknowledgements}

Dedicated to Professor Hari M Srivastava.

Received: 31 January 2013 Accepted: 19 April 2013 Published: 30 April 2013

\section{References}

1. Knobel, M, Pirota, KR: Giant magnetoimpedance: concepts and recent progress. J. Magn. Magn. Mater. 242-245, Part 1 33-40 (2002)

2. Knobel, M, Vazquez, M, Kraus, L: In: Buschow, KH (ed.) Handbook of Magnetic Materials, vol. 15 (2003)

3. Phan, M-H, Peng, H-X: Giant magnetoimpedance materials: fundamentals and applications. Prog. Mater. Sci. 53(2), $323-420(2008)$

4. Zhukov, A, Ipatov, M, Gonzalez, J, Blanco, JM, Zhukova, V: Recent advances in studies of magnetically soft amorphous microwires. J. Magn. Magn. Mater. 321(7), 822-825 (2009)

5. Zhukov, A, Zhukova, V: Magnetic Properties and Applications of Ferromagnetic Mircowires with Amorphous and Nanocrystalline Structure. Nova Science Publishers, New York (2009)

6. Chiriac, H, Ovari, TA: Amorphous glass-covered magnetic wires: preparation, properties, applications. Prog. Mater. Sci. 40(5), 333-407 (1996)

7. Mohri, K, Uchiyama, T, Shen, LP, Cai, CM, Panina, LV, Honkura, Y, Yamamoto, M: Amorphous wire and CMOS IC-based sensitive micromagnetic sensors utilizing magnetoimpedance (MI) and stress-impedance (SI) effects. IEEE Trans. Magn. 38(5), 3063-3068 (2002)

8. Mohri, K, Tsuyoshi, U, Panina, LV: Recent advances of micro magnetic sensors and sensing application. Sens. Actuators A 59(1-3), 1-8 (1997)

9. Mohri, K, Panina, LV, Uchiyama, T, Bushida, K, Noda, M: Sensitive and quick response micro-magnetic sensor utilizing magneto-impedance in Co-rich amorphous wires. IEEE Trans. Magn. 31(2), 1266-1275 (1995)

10. Panina, LV, Mohri, K: Magneto-impedance effect in amorphous wires. Appl. Phys. Lett. 65(9), 1189-1191 (1994)

11. Qin, $\mathrm{FX}$, Peng, $\mathrm{HX}$, Phan, $\mathrm{MH}$ : Wire-length effect on $\mathrm{GMI}$ in $\mathrm{CO}_{703} \mathrm{Fe}_{37} \mathrm{~B}_{10} \mathrm{Si}_{13} \mathrm{Cr}_{3}$ amorphous glass-coated microwires. Mater. Sci. Eng. 167, 129-132 (2010)

12. Kucuk, I: Multilayered perceptron neural networks to compute energy losses in magnetic cores. J. Magn. Magn. Mater. 307, 53-61 (2006)

13. Kucuk, I: Estimation of thermally stimulated current in as grown TIGaSeS layered single crystals by multilayered perceptron neural network. Expert Syst. Appl. 38, 7192-7194 (2011)

14. Kohonen, $\mathrm{T}$ : The self-organizing map. Proc. IEEE. 78(9), 1464-1480 (1990)

doi:10.1186/1029-242X-2013-216

Cite this article as: Kaya: Prediction of giant magneto-impedance effect in amorphous glass-coated micro-wires using artificial neural network. Journal of Inequalities and Applications 2013 2013:216. 